\title{
Enhancing Prediction Accuracy in PCM-Based File Prefetch by Constained Pattern Replacement Algorithm
}

\author{
Inchul Choi and Chanik Park \\ Department of Computer Science and Engineering/PRIL \\ Pohang University of Science and Technology \\ Kyungbuk, Republic of Korea \\ \{sharpic, cipark \}@postech.ac.kr
}

\begin{abstract}
In modern file systems, I/O latency is still major bottleneck of performance and predictive file prefetching is one of promising approaches that can enhance I/O performance of file system. To utilize predictive file prefetching to file system, there should be a file access pattern prediction model that can predict future file access. Partitioned Context Model(PCM) [2] is known as one of the most accurate file access pattern prediction models[3]. In order to predict longer sequence, the order of PCM must be increased. However, the prediction accuracy of PCM decreases when PCM is in high order. Careful analysis reveals that the pattern replacement algorithm in the $\mathrm{PCM}$ is the major cause in decay of the prediction accuracy. The pattern replacement algorithm destroys file access patterns without successful training of newly occurred file access patterns. We proposed the constrained pattern replacement algorithm to overcome this adverse effect by revising replacement condition. The simulation results using the DFSTrace system trace[13] show that the proposed algorithm improves prediction accuracy without any extra cost by $3.5 \%$ compared to traditional pattern replacement algorithm of $\mathrm{PCM}$ (about $40 \%$ of the accuracy bound of $7 \%$ ).
\end{abstract}

\section{Introduction}

Despite impressive advances in file system throughput resulting from technologies such as high-bandwidth networks and disk arrays, file system latency has not improved enough.In many cases, it has become worse because of the speed gaps to follow the modern processor. File system I/O remains one of the major bottlenecks against the operating system performance. Predictive file prefetching is one of promising approaches to reduce file system latency. For predictive file prefetching to be effective, however, access prediction should be accurate and far enough in advance to effectively mask out the prefetch latency.

Partitioned Context Model(PCM) is known as one of the most accurate file access pattern prediction model[3] and it can be used for sequence prediction by using a high order context model. We discovered that when we increase the 
order of the model for the prediction of file sequences, the prediction accuracy of PCM decreases. To find the cause of this effect, we analysed the file access pattern learning algorithm of PCM. The pattern replacement algorithm of PCM is found to be the one of major cause of decay of prediction accuracy in a high order PCM. The pattern replacement algorithm, which we call the unconstrained pattern replacement algorithm, of PCM can destroy the file access pattern in a partition without successful training of the newly occurred file access pattern, and it is the cause of the loss of high order file access pattern.

We propose a new replacement algorithm to overcome this adverse effect of the unconstrained pattern replacement algorithm. In the proposed replacement algorithm, when the full partition is met, the test proceeds after decaying of patterns in the partition whether the unconstrained pattern replacement algorithm deletes the same higher order nodes, especially the parent node of the newly arrived file access pattern. If true, decayed patterns in the partition is restored to its previous state to keep the state information. In this situation, the training of new pattern rely on the low order context in the other partitions. This algorithm ensures the conservation of the state information of the full partition by preventing useless loss of patterns in the full partition. Useless loss of patterns in the full partition is a major cause of decay of the prediction accuracy in high order PCM. So the proposed constrained pattern replacement algorithm enhances the high order prediction accuracy of PCM. The result of the simulation using the DFSTrace system shows that the proposed algorithm improves prediction accuracy without any extra cost by about $3.5 \%$ (about $40 \%$ of the accuracy bound of $7 \%$ between the FMOCM and the PCM), even if we increase the order to $74 \%$ of the partition size for file sequence prediction.

The remainder of this article is organized as follows. Section 2 briefly describes file access pattern prediction models. In section 3 we analysed the pattern learning algorithm of PCM and present the problem of replacement algorithm. Then we propose the new pattern replacement algorithm to enhance high order prediction accuracy of PCM. Section 4 presents our simulations and results. In section 5 we conclude the paper.

\section{Related Work}

In this section we present a brief background on the file access pattern prediction models and detail more about Partitioned Context Modeling.

\subsection{File Access Pattern Prediction Model}

File accesses follow previous patterns with a high probability. Last-successor prediction model is very simple prediction model which predicts that an access to file A will be followed by the same file that followed the last access to A, but from trace-based simulation[3] it correctly predicted $72 \%$ of access events. Another prediction model, the graph-based model is more complicated than last-successor model. The graph-based model keeps frequency counts for 
all files accessed within a window following an access to each file. However, the graph-based model sees extremely poor prediction accuracy, $40 \%$ on average as compared to the last-successor's accuracy $72 \%$. More accurate prediction model is Finite Multi-Order Context Model(FMOCM) which uses text compression technique to track file access patterns. The FMOCM has about $81 \%$ prediction accuracy and it reduced the difference between last-successor and an ideal predictor by greater than on third. But, this high prediction accuracy is achieved at the cost of significantly greater state space overhead. So, improved FMOCM which called a Partitioned Context Model(PCM) is proposed. This model addresses the state space overhead problem of FMOCM, while achieving comparable prediction accuracy with FMOCM. Next section, we detailed about Partitioned Context Modeling with Context Modeling which PCM is based on.

\subsection{Partitioned Context Modeling (PCM)}

First, we describe about context modeling. A context model $(\mathrm{CM})$ is one that uses preceding events to model the next event. For example, in the string 'object' the character ' $t$ ' is said to occur within the context 'objec'. The length of a context is termed its order. Techniques that predict using multiple contexts of varying orders(e.g.'ec', 'jec', 'bjec') are termed Multi-Order Context Models. To prevent the model from quickly growing beyond available resources, most implementations of a multi-order context model limit the highest order modeled to some finite number m, hence the term Finite Multi-Order Context Model.

A context model uses a trie, a data structure based on a tree, to efficiently store sequences of symbols. Each node in this trie contains symbol(e.g. a letter from the alphabet, or the name of a specific file). By listing the symbols contained on the path from the root to any individual node, each node represents an observed pattern.

The state space for this model is proportional to the number of nodes in this tree, which is bounded by $O\left(n^{m}\right)$, where $m$ is the highest order tracked and $n$ is number of unique files. On a normal file system the number of files can range between 10 thousand and 100 million, such space requirements are unreasonable.

To address the space requirements of FMOCM, the Partitioned Context Model is proposed. This model divides the trie into partitions, where each partition consists of a first order node and all of its descendants. The number of nodes in each partitions is limited to a static number that is a parameter of the model. The effect of these changes is to reduce the model space requirements from $O\left(n^{m}\right)$ to $O(n)$.

\section{Constrained Pattern Replacement Algorithm in PCM}

\subsection{Pitfalls of Traditional Pattern Replacement Algorithm}

FMOCM and PCM have basically the same pattern learning algorithm. However, PCM has a static partition size limit, so it can't store all of the patterns 
it has learned previously. To learn newly occurred file access pattern while preserving static partition size, PCM destroy patterns in a partition that is less probable to occur. Namely, PCM replace less probable patterns with newly occurred patterns and so pattern replacement algorithm is learning algorithm of PCM. The PCM replacement algorithm is as follows. When a new node is needed in a full partition, all node counts in the partition are divided by two (integer division), any nodes with a count of zero are cleared to make space for new nodes. If no space becomes available, the access is ignored. The pattern replacement algorithm of PCM is very simple and has no constraint when it destroys the patterns in a partition. From now we call this simple replacement algorithm as Unconstrained Pattern Replacement Algorithm. When PCM is at low order, Unconstrained Pattern Replacement Algorithm causes the model to adapt faster to new access patterns. As a result, prediction accuracy can be slightly increased. But, when PCM is in high order, the prediction accuracy of PCM decreases as the order increases. The cause of this problem reside in the difference between PCM and FMOCM, and the only difference between FMOCM and PCM is in pattern learning algorithm, the Unconstrained Pattern Replacement Algorithm. The Unconstrained Pattern Replacement Algorithm is based on the decaying of patterns in the partition and it has no constraint condition for pattern destroying.

As the order of PCM increases, the number of pattern decaying increases also because the partition size is limited to a static number. An increase in the number of pattern decaying can result in lower average node count. When the average node count in partition is low, the Unconstrained Pattern Replacement Algorithm can cause problems. Figure 1 describes the trie structre trained by sequence 'ABCABEF' and situation that the new access to ' $G$ ' is occured. The Unconstrained Pattern Replacement Algorithm can cause problems from the situation like in Figure 1.

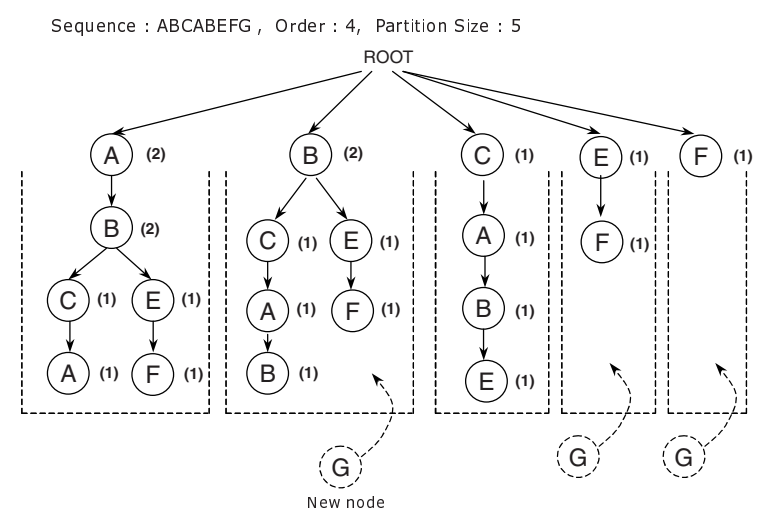

Fig. 1. Example trie before Unconstrained Pattern Replacement for the sequence ABCABEFG 
In Figure 1, the new node ' $G$ ' which represents the pattern 'BEFG' is about to added to second partition, but the partition is full, so pattern decaying (integer division by 2) occurs and zero count nodes are cleared. Figure 2 shows situation after pattern decaying in the second partition. After Unconstrained Pattern Replacement in the second partition, the number of nodes are severely dropped and the parent node of new node ' $G$ ' is cleared out. To store the pattern 'BEFG' by adding new node ' $G$ ', nodes represents 'BEF' should not be cleared out. However, when we use the Unconstrained Pattern Replacement Algorithm, this cannot be achieved. As a result, patterns are lost without learning the newly occurred pattern. Situations similar to Figure 1 are more often met when the order of model is high, because high order means low average node count in the partitions. So, if we overcome this adverse effect, we can expect increase of prediction accuracy in high order PCM.

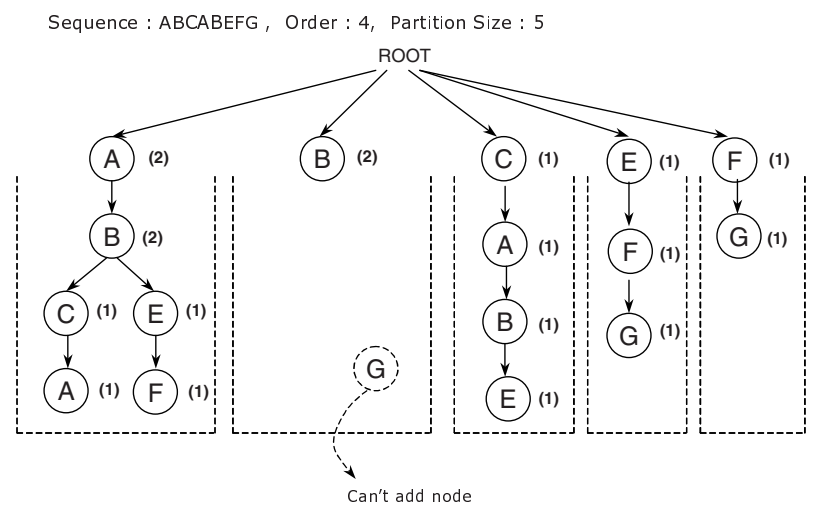

Fig. 2. Example trie after Unconstrained Pattern Replacement for the sequence ABCABEFG

\subsection{Constrained Pattern Replacement Algorithm}

To overcome the adverse effect of Unconstrained Pattern Replacement Algorithm of PCM in high order, we propose a new replacement algorithm which has constraint condition for pattern decaying. We call this new replacement algorithm as Constrained Pattern Replacement Algorithm. The Constrained Pattern Replacement Algorithm check whether the parent node of new arrived file access pattern is deleted after decaying of patterns. And if this is true, restore the partition to its previous state. 


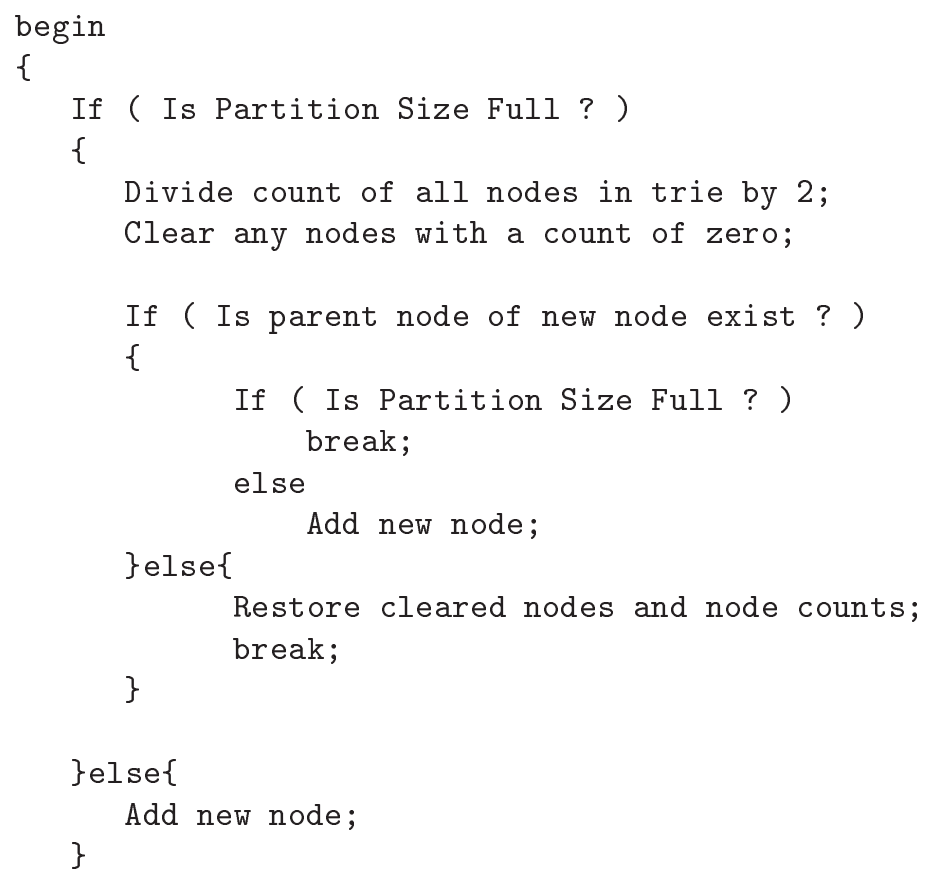

\}until ( number of new nodes to add == 0 )

The Constrained Pattern Replacement Algorithm can prevent the useless decaying of patterns in partition without learning of newly occurred pattern. For example, if we use Constrained Pattern Replacement Algorithm in situation of Figure 1, we decay patterns in the partition and have a situation like Figure 2. Then we check whether the parent node of newly arrived pattern is cleared out and find out it is true in second partition. So, patterns in the second partition is restored to their previous state. Figure 3 shows the trie structure after Constrained Pattern Replacement Algorithm is applied to Figure 1.

From Figure 3, we can know that the Constrained Pattern Replacement Algorithm keeps the state information of second partition to prevent the useless decaying of patterns.

\section{Simulation Results}

To compare the prediction accuracy of PCM with our Improved PCM(PCM with Constrained Pattern Replacement Algorithm), we used trace data collected with the DFSTrace system. For simulation we selected the workload of barber machine which represents the characteristics of file server workload. The machine barber was also a server with the highest rate of system calls per second. For comparison 


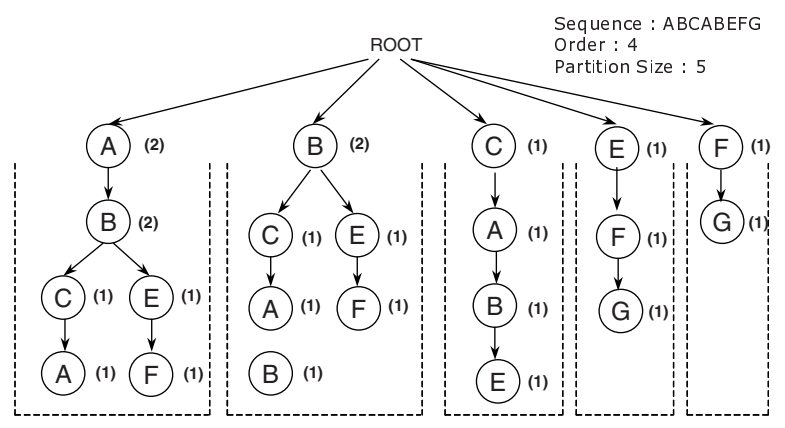

Fig. 3. Example trie after Constrained Pattern Replacement for the sequence ABCABEFG

purposes, we define measure Useful Prediction: $U_{p}$ and Prediction Accuracy: $P_{a}$ as follows.

$$
\begin{aligned}
U_{p} & =\frac{\text { Successful prediction count }}{\text { Total file access count }} \\
P_{a} & =\frac{\text { Successful prediction count }}{\text { Total prediction count }}
\end{aligned}
$$

$U_{p}$ represents the usefulness of prediction and $P_{a}$ represents the prediction efficiency. From above definition we compare the PCM with Improved PCM by difference of $U_{p}$ and $P_{a}$. Definition of $D_{U}$ and $D_{P}$ are as follows.

$$
\begin{aligned}
& D_{U}=U_{p(\text { Improved } P C M)}-U_{p(P C M)} \\
& D_{P}=P_{a(\text { Improved } P C M)}-P_{a(P C M)}
\end{aligned}
$$

We simulated PCM and Improved PCM with Partition Size 32,48 and predict most probable file from the longest context. To comparison purpose, we measure $D_{U}, D_{p}$ with increasing order. In Figure $4 D_{U}$ and $D_{p}$ is positive in high order and has maximum value $3.5 \%$ which means Improved PCM has $3.5 \%$ better $U_{p}$ and $P_{a}$. The result of partition size 48 in Figure 5 is also similar. Considering the maximum difference of $U_{p}$ between FMOCM and PCM is $7 \%$, improvement in prediction accuracy is corresponds to approximately $40 \%$.

Figure 6 and Figure 7 compare $U_{p}$ between traditional pattern replacement algorithm and constrained pattern replacement algorithm. As we can see from Figure 6 and Figure 7, the Constrained Replacement Algorithm improved prediction accuracy of PCM in high order.

\section{Conclusion}

In this paper we proposed a new replacement algorithm to enhance the prediction accuracy of PCM in high order. We analysed pattern learning algorithm 


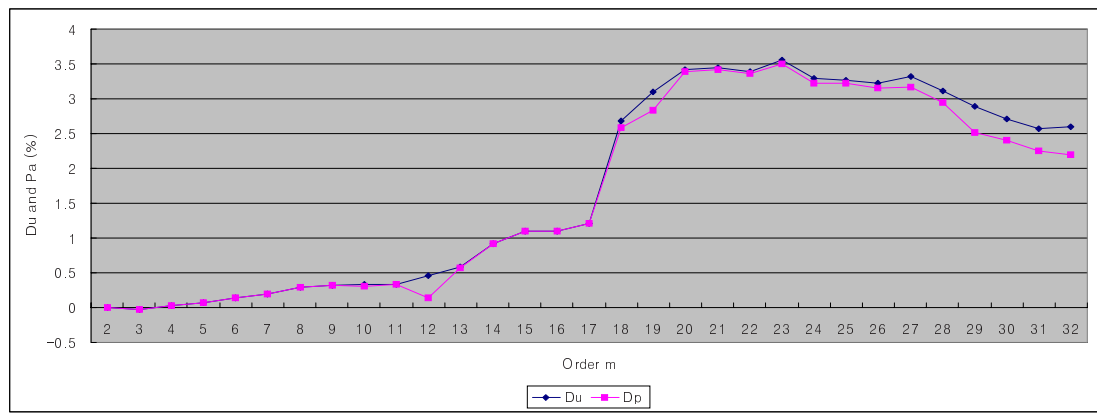

Fig. 4. $D_{U}$ and $D_{p}$ when Partition Size is 32

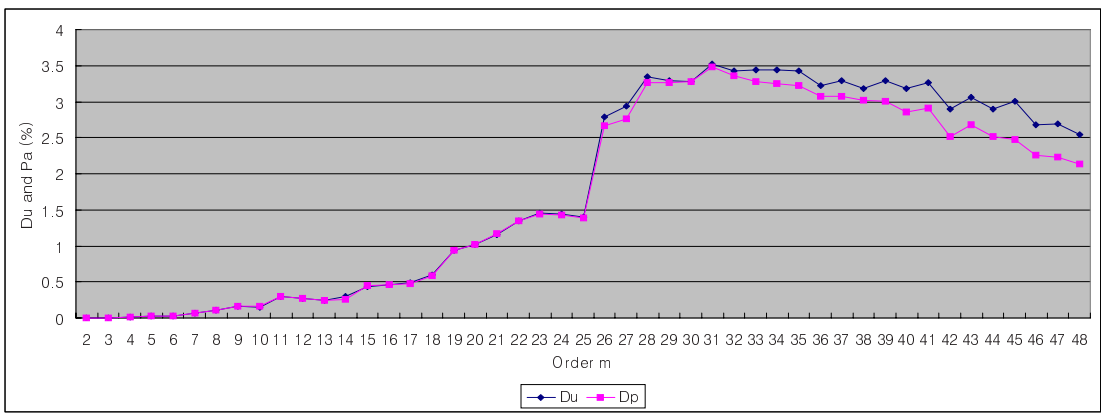

Fig. 5. $D_{U}$ and $D_{p}$ when Partition Size is 48

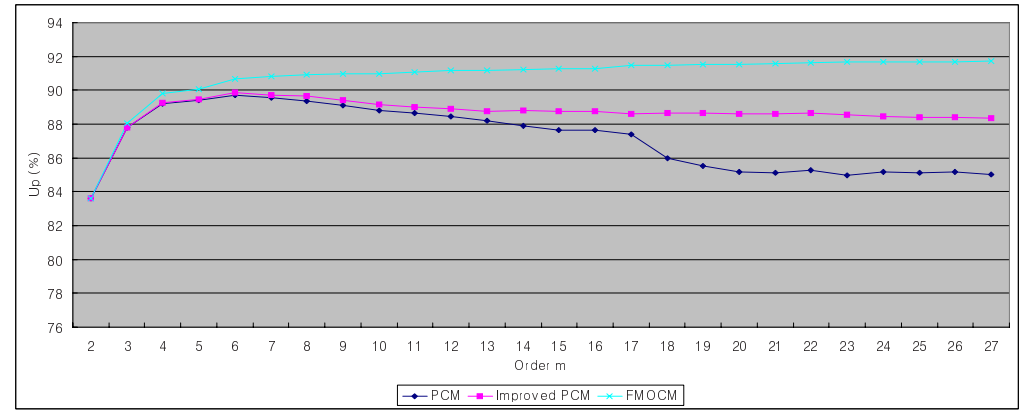

Fig. 6. Comparison of Useful Prediction between traditional pattern replacement algorithm and constrained pattern replacement algorithm (Useful Prediction vs. order of PCM when partition size is 32 ) 


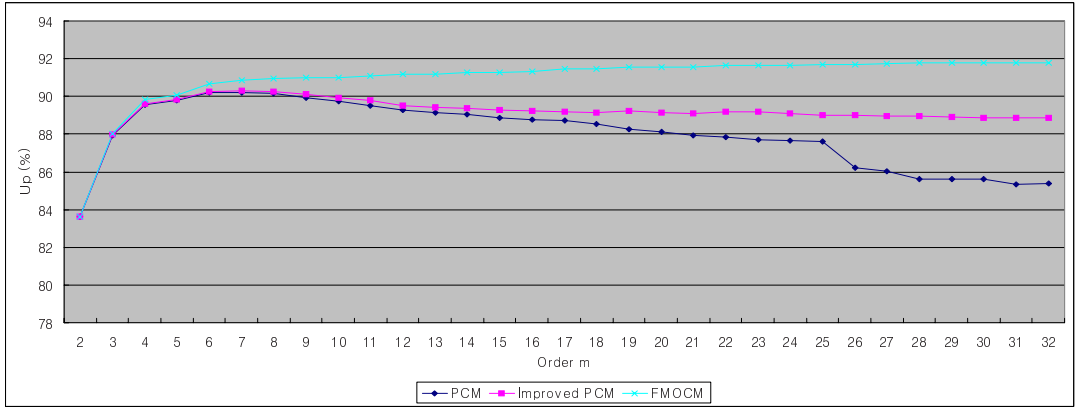

Fig. 7. Comparison of Useful Prediction between traditional pattern replacement algorithm and constrained pattern replacement algorithm (Useful Prediction vs. order of PCM when partitioin size is 48)

of PCM and discovered the cause of decrease of prediction accuracy in high order. We also proposed Constrained Pattern Replacement Algorithm to prevent useless loss of patterns in partition. Consequently, we enhanced the prediction accuracy of PCM in high order. From the trace-based simulation results, using DFSTrace system trace, we can see Improved PCM which uses Constrained Pattern Replacement Algorithm has a better prediction performance than PCM. Improved PCM has maximum 3.5\% improved $U_{p}$ and $P_{a}$ in high order, which corresponds to $40 \%$ improvement compared to maximum $7 \%$ difference between FMOCM and PCM.

\section{Acknowledgement.}

The authors would like to thank the Ministry of Education of Korea for its financial support toward the Electrical and Computer Engineering Division at POSTECH through its BK21 program. This research was also supported in part by HY-SDR IT Research Center.

\section{References}

1. T. M. Kroeger and D. D. E. Long, "Design and implementation of a predictive file prefetching algorithm," in Proceedigs of the 2001 USENIX Annual Technical Conference, Boston, MA, Jan. 2001.

2. T. M. Kroeger and D. D. E. Long, "Predicting file system actions from prior events," in Proceedigs of the USENIX 1996 Annual Technical Conference, pp. 319328, USENIX, Jan. 1996.

3. T. M. Kroeger and D. D. E. Long, "The case for efficient file access pattern modeling." in IEEE Proceedings of the Seventh Workshop on Hot Topics in Operating Systems (HotOS-VII), Rio Rico, Arizona, Mar. 1999.

4. T. M. Kroeger and D. D. E. Long, "Predicting file system actions from prior events," Master's thesis, University of California, at Santa Cruz, 1997. 
5. G. Kuenning, "The design of the seer predictive caching system," in Workshop on Mobile computing Systems and Applications, pp.37-43, IEEE Computer Society, Dec. 1994.

6. H. Lei and D. Duchamp, "An analytical approach to file prefetching," in Proceedigs of USENIX 1997 annual Technical Conference, USENIX, Jan. 1997.

7. V. N. Padmanabhan and J. C. Mogul, "Using Predictive Prefetching to Improve World Wide Web Latency," in Proceedings of the 1996 SIGCOMM, ACM, July 1996.

8. J. S. Vitter and P. Krishnan, "Optimal prefetching via data compression," Journal of the ACM, vol. 43, pp. 771-793, Sep. 1996.

9. K. M. Curewitz, P. Krishnan, and J. S. Vitter, "Practical prefetching via data compression," in Proceedings of the 1993 ACM SIGMOD International Conference on Management of Data (SIGMOD '93), pp. 257-266, Washington, D. C., May 1993.

10. J. Griffioen and R. Appleton, "Reducing file system latency using a predictive approach," in USENIX Summer Technical Conference, pp. 197-207, June 1994.

11. T. M. Madhyastha and D. A. Reed, "Input/output access pattern classification using hidden Markov models," in Proceedings of the Fifth Workshop on Inpu/Output in Parallel and Distributed Systems, (SanJose, CA), pp. 57-67, ACM Press, Nov. 1997.

12. A. Amer and D. D. E. Long, "Noah: Low-cost file access prediction through pairs, " in Proceedings of 20th IEEE International Performance, Computing, and Communications Conference (IPCCC 2001), pp. 27-33, Phoenix, Arizona, Apr. 2001.

13. L. Mummert and M. Satyanarayanan, "Long term distributed file reference tracing: Implementation and experience, " Sofware - Practice and Experience (SPE), 26(6):705-736, June 1996.

14. Ahmed Amer, Darrell D. E. Long, and Randal C. Burns, "Group-Based Management of Distributed File Caches," The IEEE International Conference on Distributed Computing Systems (ICDCS 2002), Vienna, Austria: July 2002.

15. Ahmed Amer, Darrell D. E. Long, Jehan-Francois Paris, and Randal C. Burns, "File Access Prediction with Adjustable Accuracy," Proceedings of the IEEE International Performance Conference on Computers and Communi-cation (IPCCC 2002), Phoenix, Arizona: Apr. 2002.

16. J.G. Cleary, W. Teahan, and I.H. Witten, "Unbounded length contexts for PPM, " J.A. Storer and M. Cohn (eds.), Proc. IEEE Data Compression Conference, Snowbird, Utah, 1995. 